\title{
Les risques du métier. Partie 3
}

Daniel Cefaï et Valérie Amiraux

\section{(2) OpenEdition}

\section{Journals}

\section{Édition électronique}

URL : http://journals.openedition.org/conflits/835

DOI : 10.4000/conflits.835

ISSN : $1777-5345$

Éditeur :

CCLS - Centre d'études sur les conflits lilberté et sécurité, L'Harmattan

\section{Édition imprimée}

Date de publication : 1 septembre 2002

ISBN : 2-7475-3432-4

ISSN : 1157-996X

\section{Référence électronique}

Daniel Cefaï et Valérie Amiraux, «Les risques du métier. Partie 3 », Cultures \& Conflits [En ligne], 47 |

automne 2002, mis en ligne le 29 avril 2003, consulté le 30 mars 2021. URL : http://

journals.openedition.org/conflits/835; DOI : https://doi.org/10.4000/conflits.835

Ce document a été généré automatiquement le 30 mars 2021.

Creative Commons License 


\title{
Les risques du métier. Partie 3
}

\author{
Daniel Cefaï et Valérie Amiraux
}

\section{Anthropologues militants}

L'engagement pratique des anthropologues n'est pas nouveau. Le secteur de l'«anthropologie appliquée» qui s'est développé aux Etats-Unis dès les années quarante $^{1}$ visait explicitement à être en prise sur des programmes de politiques publiques ou à améliorer les relations humaines en entreprise. A cette figure de l' " ingénieur des facteurs humains $»^{2}$ a succédé celle du militant des human rights de l'advocacy anthropology ${ }^{3}$, qui a donné naissance à nombre d'observatoires des droits de l'homme. Aujourd'hui, Nancy Scheper-Hughes synthétise un certain nombre de propositions pour une " anthropologie militante $»^{4}$. Elle a coordonné des projets sur la politique de l'enfance ${ }^{5}$, après avoir de longues années enquêté sur les formes de violence quotidienne au Brésil ${ }^{6}$. Pourquoi des mères laissent-elles mourir leurs enfants en invoquant avec fatalité leur "nervosisme » et en négligeant de leur donner aucun soin, sans compassion? Pourquoi les massacres des enfants de rue et des sans-abri par les escadrons de la mort ou les assassinats des sans-terre par les milices armées des propriétaires terriens, ont-ils été si longtemps ignorés? N. Scheper-Hughes critique l'empirisme positiviste et le relativisme post-moderniste comme deux manières de court-circuiter les questions éthiques et politiques. L'enquête selon elle doit inclure un moment de solidarité et de reconnaissance avec les enquêtés et peut être un moyen d'augmenter leur capacité d'autonomie et de résistance.

Sans que la chose prenne toujours un tour aussi militant, la corporation des anthropologues est de plus en plus ramenée à la dimension éthique et politique de ses activités. Elle a été bouleversée par différentes affaires, comme celle de $\mathrm{C}$. Turnbull, accusé par F. Barth ${ }^{7}$ d'avoir observé et décrit dans The Mountain People l'agonie d'un peuple Pygmée sans intervenir, ou celle toute récente mettant en cause N. Chagnon et T. Asch, auteurs de monographies et de documentaires sur les Yanomami, et soupçonnés d'avoir procédé avec l'aide d'un généticien, J. Neel, à l'insémination de la rougeole en vue de prouver des théories eugénistes sur une population primitive ${ }^{8}$. Elle est ébranlée par les faits de bio-piratage de la pharmacopée indigène ou de leur patrimoine génétique par des firmes internationales ou des laboratoires scientifiques. Sur le terrain, les anthropologues sont saisis par l'urgence d'agir et de venir en aide à 
leurs «sujets». Un amazoniste comme Bruce Albert perçoit son travail de terrain comme une forme de "participation observante ", où il assume son rôle d'expert pour des banques multilatérales et des agences onusiennes, où il pourvoit des données et des arguments aux commissions qui préparent des lois indigènes, où il élabore des matériaux pédagogiques et didactiques destinés aux agents de santé, aux professeurs et aux médecins et où, à l'occasion, il sert d'expert pour des cas d'assassinat ou de spoliation ${ }^{9}$. Alexandro Surrales doit s'impliquer au jour le jour dans des campagnes de vaccination et de dépistage de maladies, assister les chefs indiens dans leur travail de coordination des tribus dispersées dans la forêt, prendre part sur le terrain à des opérations de démarcation des territoires indigènes, parfois œuvrer comme médiateur entre propriétaires terriens, agences gouvernementales et groupes indigènes et remplir à l'occasion le rôle de secrétaire de l'organisation indigène à Lima. La part de la recherche est inextricablement encastrée dans celle de l'intervention, comme en témoigne Bernard Moizo, qui agit successivement comme conseiller d'une organisation aborigène locale et expert pour le gouvernement australien ${ }^{10}$. Avec le recul, certains le confessent, cette position de traducteur, de médiateur et d'avocat pour le compte de la population étudiée est un gage de maintien de l'accès au terrain et de qualité des informations recueillies. Mais cet intérêt scientifique est indissociable d'une défense de la survie, d'une exigence de justice et d'une lutte pour les droits des «sujets » de l'enquête.

Expertise et organisations internationales

Les conditions de la commande et les circonstances de la réception de l'enquête doivent donc être anticipées par l'enquêteur. Cela s'impose dorénavant d'autant plus qu'une coopération plus ou moins stable s'engage entre des experts issus des universités ou de centres de recherche et les institutions publiques - sous des formes variées qui vont de la simple consultance pour une ONG ou une fondation, à la contractualisation par une institution comme le FMI ou la Banque mondiale. Une logique de marché domine le champ de l'expertise et permet à certains de s'assurer des rentes de position, en partie $\mathrm{du}$ fait du déplacement de la compétence d'expert de la haute fonction publique vers les milieux universitaires ${ }^{11}$. Mais l'émergence d'un nouveau marché, avec ses formations et ses compétences, a des effets politiques. Le rôle joué par des spécialistes de zones géographiques dans la gestion des programmes internationaux de promotion des droits de l'homme et d'aide à la démocratisation illustre les mécanismes de participation des experts à la mise en place de projets politiques ${ }^{12} \mathrm{~N}$. Guilhot analyse par exemple le déplacement vers les institutions étatiques d'un activisme des droits de l'homme et le développement d'une expertise militante. La microsociologie des itinéraires biographiques des principaux « inventeurs » de la transition démocratique auprès de la Banque Mondiale dans les années 1980 révèle ainsi d'anciens militants issus des Latin American Studies, opposés au soutien de la même Banque aux régimes autoritaires dans les années $1970^{13}$.

Les experts organisent la circulation de leurs concepts et de leurs modèles entre les différents contextes d'expérience et d'activité auxquels ils prennent part. D. Bigo a ainsi analysé les conditions du succès de la thèse de $\mathrm{S}$. Huntington sur le clash des civilisations dans la discipline des relations internationales et auprès des responsables politiques américains. "Ces discours qui se présentent comme académiques visent avant tout à reconvertir le capital spécifique de la 'gestion de la menace' que détiennent les professionnels de la sécurité et à redonner aux spécialistes de relations internationales leur place de conseillers de ces professionnels $»^{14}$. La représentation de 
l'incertitude qui règne dans l'environnement international a motivé le diagnostic en $\mathrm{R}$. I. de la nécessité pour les acteurs politiques de renouveler leurs sources d'inspiration et de diversifier leurs sources de conseil ${ }^{15}$. Cet appel d'air a permis à des réseaux de professionnels de revendiquer avec autorité une expertise en matière de politique étrangère $\mathrm{e}^{16}$. Les exemples sont nombreux de cette connexion des relations internationales avec le pouvoir politique ${ }^{17}$. Elle a mis en jeu d'autres disciplines, non sans scandales. Aux Etats-Unis, dès les années 1950, les agences gouvernementales chargées de la contre-insurrection ont entrepris de téléguider d'énormes projets anthropologiques ${ }^{18}$ Le Himalayan Border Countries Project ${ }^{19}$, le Projet Camelot en Amérique latine ${ }^{20}$ et le Projet Agile en Thaïlande ${ }^{21}$ étaient directement financés par l'US Defense Department, qui avait publié à l'adresse des militaires un premier volume d'informations sur les groupes minoritaires du Nord Vietnam ${ }^{22}$. Et une bonne part des projets de recherche sur les " caractères nationaux » comme le Russian Research Center de C. Kluckhohn à Harvard ou le Human Relations Area Files de G. P. Murdock à Yale ou sur les conditions du développement politique, dont le prototype reste Civic Culture de G. Almond et S. Verba, avaient des visées de fondation d'un nouvel ordre international pour le gouvernement américain ${ }^{23}$.

Les risques du métier de chercheur, pour ce qui concerne le travail d'enquête, sont multiples et ne se limitent pas aux dangers physiques. En organisant cet article en deux volets, nous avions pour objectif principal de montrer quelques-unes des difficultés du travail d'enquête dans la continuité d'un processus allant du recueil des données à leur utilisation dans des cadres non scientifiques. De la sorte, nous avons fait incursion du côté des questionnements éthiques et politiques qui se posent à d'autres disciplines que l'anthropologie et la sociologie.

Le point aujourd'hui le plus brûlant est celui du contrôle des résultats finaux par les enquêtés eux-mêmes. Les arènes d'information, de consultation et de délibération qui se sont multipliées ces dernières années font de plus en plus appel à la compétence des experts, favorisent la diffusion des rapports d'enquête auprès d'interlocuteurs qui, jusqu'à présent, n'y avaient pas accès. Les conditions de la recherche ont changé. Il n'est pas rare qu'un même terrain soit investi par plusieurs chercheurs de disciplines différentes qui proposent des lectures non nécessairement convergentes. Se déploient alors d'autres enjeux, inédits, en termes de concurrence pour gagner la confiance des uns, faire remonter les revendications des autres. Plus que jamais, il semble que l'inconfort du chercheur, initialement lié aux conditions de son accès et de son séjour "sur place ", aux attaches et aux ententes nées de la rencontre avec les enquêtés et aux dilemmes, contradictions et paradoxes propres à cet engagement se soit étendu au moment de l'expertise et de sa réception.

L'engagement $\mathrm{du}$ chercheur dans son objet est donc toujours problématique. Implications personnelles, intérêts professionnels, convictions militantes: les raisons de la recherche sont souvent inextricables. Le vieux triptyque weberien de l'appétit d'argent, de pouvoir et de réputation ne suffit pas à tout expliquer. Le sentiment de justice, l'activité de médiation, le devoir de mémoire ou le désir de vérité sont des motivations et des justifications qui président tout autant au cours de l'enquête. Quelque soit le cas de figure, l'évaluation de la validité des connaissances en histoire et en sciences sociales est indissociable de la question de qui apprend quoi, où et quand, pourquoi et comment, au bénéfice de qui et au détriment de qui, en vue de quels usages et avec quelles conséquences. Le compte-rendu des conditions de commande et de 
réception de l'enquête, des engagements problématiques de l'enquêteur et de ses dilemmes éthiques et politiques constitue désormais un élément de la réflexivité du métier de chercheur.

\section{NOTES}

1. La création de la revue Applied Anthropology date de 1941. Elle est dirigée à

l'époque par des proches de W. Lloyd Warner, Conrad Arensberg et Elliott Chapple, qui passent le témoin en 1948 à William F. Whyte, l'auteur de Street Corner Society.

2. . Whyte W. F., Learning From the Field : A Guide From Experience, Beverly Hills, Sage, 1984.

3. . Wright R., « Anthropological Presuppositions of Indigenous Advocacy », Annual Review of Anthropology, 1988, 17, pp. 365-390.

4. . D'Andrade R., Scheper-Hughes N., « Objectivity and Militancy : A Debate (1. Moral Models in Anthropology ; 2. The Primacy of the Ethical) », Current Anthropology, June 1995, 36, 3, pp. 399-440.

5. . Scheper-Hughes N. (ed.), Child Survival : Anthropological Perspectives on the Treatment and Maltreatment of Children, D. Reidl, Culture, Illness, and Healing Series, 1987 et Scheper-Hughes N., Sargent C. (eds.), Small Wars : The Cultural Politics of Childhood, University of California Press, 1998.

6. . Scheper-Hughes N., Death Without Weeping : The Violence of Everyday Life in Brazil, Berkeley, Los Angeles, University of California Press, 1992.

7. . Barth F., « On Responsibility and Humanity : Calling a Colleague to Account », Current Anthropology, 1974, 5, 1, pp. 99-102.

8. . Tierney P., Darkness in El Dorado : How Scientists and Journalists Devastated the Amazon, New York/London, Norton, 2001.

9. . Albert B., « Situation ethnographique et mouvements ethniques. Réflexions sur le terrain post-malinowskien », in Agier M. (ed.), Anthropologues en dangers, op. cit., 1997, pp. 75-88.

10. . Moizo B., «L'anthropologie aboriginaliste : de l'application à la fiction », in M. Agier (dir.), op. cit., pp. 65-74, ici p. 65.

11. . Voir le recensement par L. Tanguy des missions sur l'éducation et l'enseignement en France : Tanguy L., « Le sociologue et l'expert : une analyse de cas », Sociologie du travail, 1995, n³, pp. 457-477.

12. . Les modifications de l'approche du développement par la Banque mondiale dans les années 1980 dix en sont un exemple : les ONG, devenues des alliées, adoptent le discours du développement durable.

13. . Guilhot N., «Les professionnels de la démocratie : Logiques militantes et logiques savantes dans le nouvel internationalisme américain ", in Actes de la recherche en sciences sociales, septembre 2001, 139, pp. 53-65, ici p. 59.

14. . Bigo D., « Grands débats dans un Petit Monde. Les débats en relations internationales et leur lien avec le monde de la sécurité », Cultures \& Conflits, 19/20, Paris, L'Harmattan, 1995, pp. 7-48, ici p. 10. 
15. Sur les « communautés épistémiques » : Haas P., « Introduction », International Organization, 1992, 49, 1 et Haas P., When Knowledge is Power, Berkeley, University of California Press, 1990.

16. . Goldstein J., Keohane R. O., Ideas and Foreign Policy : Beliefs, Institutions, and Political Change, Ithaca/New York, Cornell University Press, 1993.

17. . Ailleurs que dans le champ de la sécurité, les agences gouvernementales, les fondations, les think tanks et les organisations internationales ont loué les services de Henry Kissinger, Joseph Nye, David Mitrany, John Ruggie ou Francis Fukuyama.

18. . G. Condominas dénonce très tôt les chercheurs qui travaillent comme informateurs de l'anti-guérilla en Asie du Sud-Est : Condominas G., « Ethics and Comfort : An Ethnographer's View of his Profession ", Distinguished Lecture 1972 de l'AAA, publié dans Annual Report of the American Anthropological Association, April 1973, pp. 1-17.

19. . Berreman G., " Academic Colonialism : Not So Innocent Abroad », in T. Weaver (ed.), To See Ourselves : Anthropology and Modern Social Issues, Glenview, IL, Scott, Foresman, 1973, pp. 152-156.

20. . Horowitz I. (ed.), The Rise and the Fall of Project Camelot : Studies in the Relationship Between Social Science and Practical Politics, Cambridge, MIT Press, 1967. 21. . Wolf E., « American Anthropologists and American Society », in D. Hymes (ed.), Reinventing Anthropology, New York, Pantheon, 1972, pp. 251-263.

22. . Kensington Office of the American Institutes for Research, Minority Groups in North Vietnam, Ethnographic Study Series, Washington DC, US Government Printing Office, 1972.

23. . Les Area Studies avaient une vocation presque explicitement stratégique.

INDEX

Mots-clés : risques, sociologie 